\title{
Highlight
}

\section{Directed Evolution of Iridium-Substituted Myoglobin Affords Versatile Artificial Metalloenzymes for Enantioselective C-C Bond-Forming Reactions}

Thomas R. Ward

\author{
Department of Chemistry \\ University of Basel, \\ Spitalstrasse 51 \\ CH-4051 Basel, Switzerland \\ email: thomas.ward@unibas.ch
}

\begin{abstract}
The group of Hartwig and coworkers recently reported on a versatile metal-substitution strategy applied to natural heme proteins that beautifully complements the more traditional approaches pursued for the creation of artificial metalloenzymes. Herein we highlight the novelty of this approach and set it into the broad context of artificial metalloenzymes.
\end{abstract}

In the past decade, artificial metalloenzymes (ArMs) have attracted increasing attention as alternative to the more traditional homogeneous catalysts and enzymes. ${ }^{[1,2]}$ Such ArMs result from the incorporation of an abiotic metal cofactor within a host protein. Until recently, three complementary strategies had successfully been pursued for the assembly of ArMs: i) Covalent anchoring whereby the ligand bound to the metal is covalently linked to the protein (Scheme 1a) ${ }^{[3]}$ ii) Supramolecular anchoring which relies on an high affinity interaction between a host protein and its guest (or inhibitor) (Scheme 1b) ${ }^{[4]}$ and iii) Dative anchoring whereby amino acid side-chains bind and activate a metal (Scheme 1c).[5] The group of Hartwig and coworkers recently reported on a versatile metal-substitution strategy applied to natural heme proteins that beautifully complements these three approaches. ${ }^{[6]}$

ArMs combine attractive features of homogeneous catalysts and enzymes. The cofactor can contain any element of the periodic table, thus significantly expanding the accessible reaction repertoire of ArMs as compared to natural biocatalysts. Furthermore, both chemical- and genetic optimization schemes have successfully been applied to improve the performance of ArMs. ${ }^{[1,2}$ However, the coinage metals, so dear to the organometallic community, are frequently inhibited by various cellular components, including glutathione. As a consequence, the host proteins must be (partially) purified prior to the addition of the abiotic cofactor, thus imposing a significant bottleneck towards directed evolution schemes that typically rely on screening large libraries of mutants. 
Capitalizing on the planar structure of heme (iron-protoporphyrin IX Fe-PIX hereafter), Watanabe, Lu and Hayashi have substituted this versatile cofactor by a variety of planar polydentate analogs or heme-derivatives to afford ArMs for a variety of transformations. In these strategies however, most of the optimization effort was focused on chemical modification of the cofactor rather than directed evolution of the host protein Scheme 2a. ${ }^{[7]}$

a)
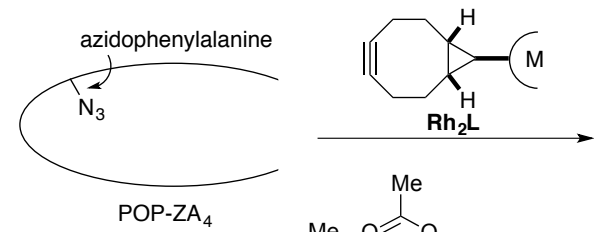

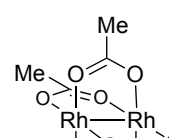

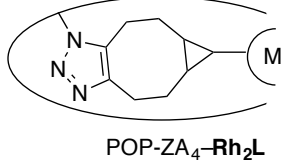

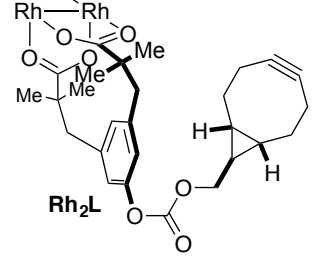<smiles>C=Cc1ccccc1</smiles>

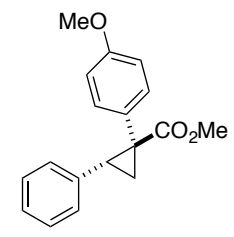

POP-ZA 4 : E104A-F146A-K199A D202A-S477Z er $69: 31,29 \%$ yield

evolved POP-ZA 104A-F146A-K199A-D202A-S477Z-L328H-G99F-G594F er $96: 4,74 \%$ yield

b)

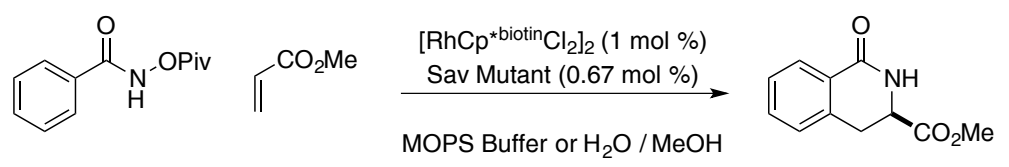

\begin{tabular}{cccc}
\hline Entry & Sav Mutant & Conversion (\%) & er \\
\hline 1 & - & $<5$ & - \\
2 & WT & $<5$ & - \\
3 & N118K-K121E & 99 & $82: 18$ \\
4 & S112Y-K121E & 95 & $91: 9$ \\
\hline \multicolumn{4}{l}{ Sav = streptavidin }
\end{tabular}

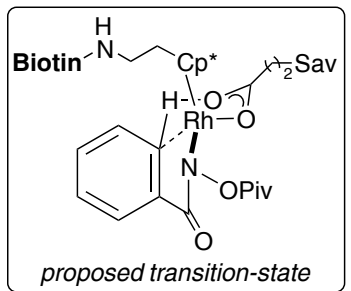

c)

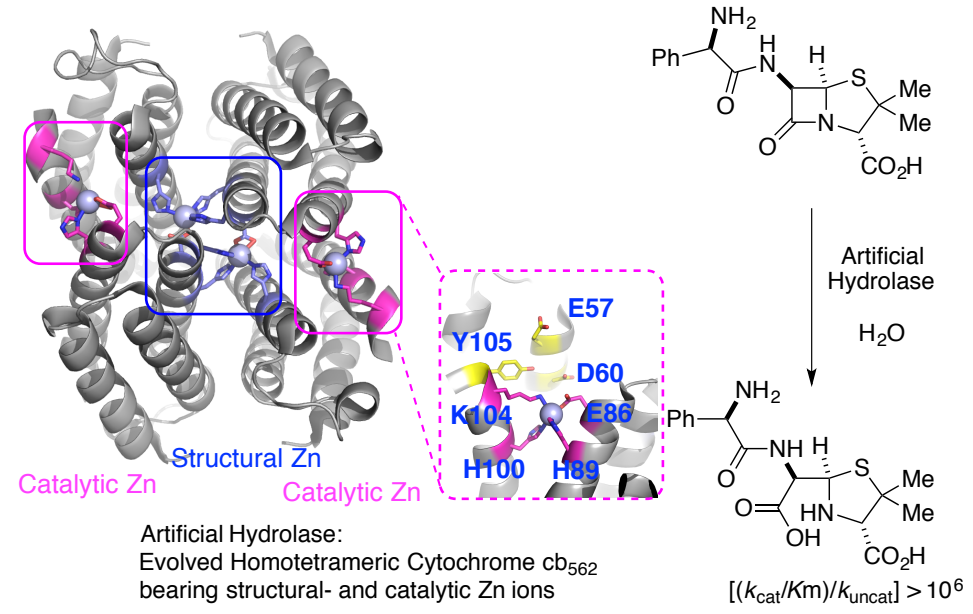

Scheme 1 Selected examples of directed evolution of ArMs based upon: covalent anchoring to azidophenylalanine engineered into Propyl OligoPeptidase (POP-ZA $\mathrm{Z}_{4}$ ) for enantioselective cyclopropanation a); supramolecular anchoring based on the biotin-streptavidin technology for 
enantioselective intermolecular $\mathrm{C}-\mathrm{H}$ activation b) and dative anchoring of zinc in engineered cytochrome $\mathrm{cb}_{562}$ for hydrolytic reactions $\mathrm{c}$ ). (Adapted from ref. 2 with permission from Wiley)

Inspired by the isolobal analogy between the $\{\mathrm{Fe}=0\}$ - and the iron-carbene or nitrene fragments, the groups of Arnold and later of Fasan repurposed cytochrome P450s and myoglobin $(\mathrm{Mb})$ to catalyse a variety of abiotic transformations: enantioselective cyclopropanation, $[9,10]$ nitrene insertion etc. Scheme 2 b, c. ${ }^{[2,8]}$ In stark contrast to ArMs, such repurposed enzymes are fully compatible with cellular components and can thus be screened and evolved in vivo or in cell lysates. This elegant strategy is thus only limited by the intrinsic reactivity of the Fe-PIX cofactor, augmented by the fine-tuning achieved via genetic means.

a)

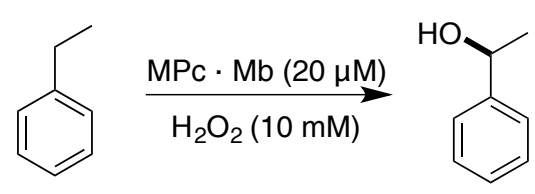

$8 \mathrm{mM} \quad \mathrm{M}=\mathrm{Mn}(\mathrm{III}) 13 \mathrm{TON} 57: 43$ er

$\mathrm{M}=\mathrm{Fe}(\mathrm{III}) \quad 0 \mathrm{TON}$

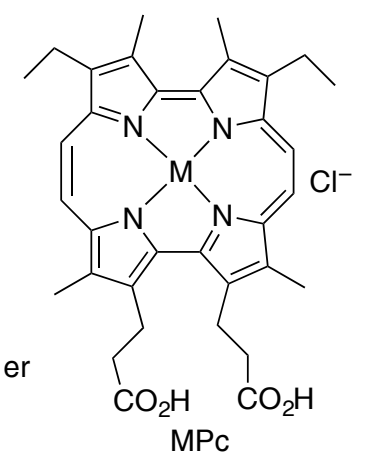

b)

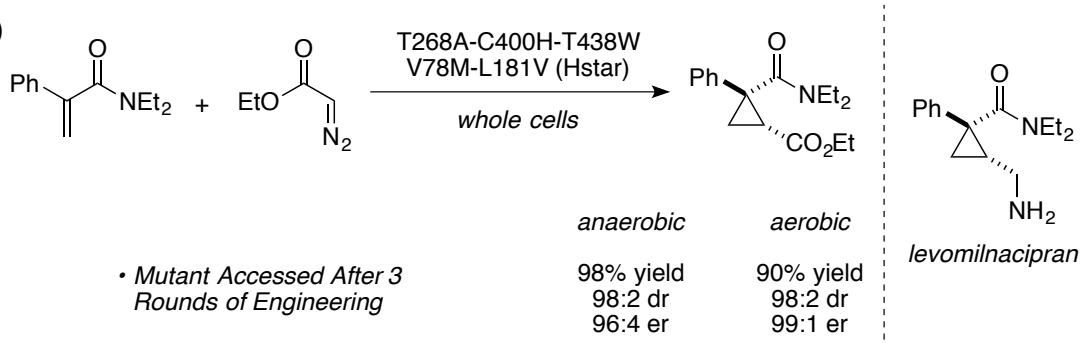

c)
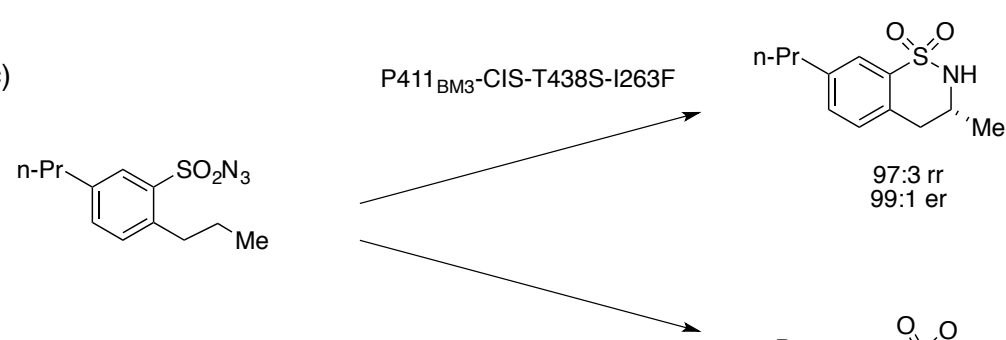

P411 ${ }_{\text {BM3 }}-$ F87A-T268A

$\mathrm{P} 41_{\mathrm{BM}}$-CIS-T438S-I263F = V78A, F87V, P142S, T175I, A184V, S226R, H236Q, E252G, I263F, T268A, A290V, L353V, I366V, C400S, T438S, E442K.

$$
97: 3 \mathrm{rr}
$$

$$
\text { 99:1 er }
$$

d)

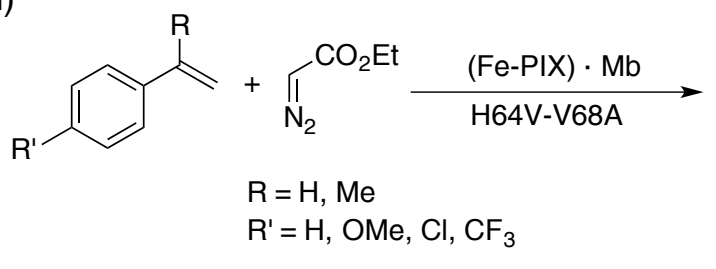<smiles>[R]OC(=O)[C@H]1C[C@@]1([R])c1ccc([R])cc1</smiles>

- up to 46800 TON

- up to 1000:1 dr

- up to 1000:1 er

Scheme 2 Repurposing heme-containing proteins for novel transformations: C-H hydroxylation relying on porphycene-reconstituted myoglobin $\mathrm{MPc} \cdot \mathrm{Mb}$ ); cyclopropanation for the synthesis of a levomilnacipran precursor relying on cytochrome $\mathrm{P}_{450_{\mathrm{BM}}}$ bearing an axial histidine $\mathrm{C} 400 \mathrm{H}$ 
b) and regioselective nitrene insertion relying on cytochrome $\mathrm{P}^{4} 11_{\text {вмз }}$ bearing an axial serine C400S c); cyclopropanation of styrene derivatives based on myoglobin mutants d) (Adapted from ref. 2 with permission from Wiley).

To capitalize on the versatile chemistry of precious metal porphyrins, the Hartwig group recently reported on a metal-substitution strategy applied to heme-proteins. ${ }^{[6]}$

Substitution of iron in protoporphyrin IX (Fe-PIX) by a variety of metals (M-PIX) allowed them to create and evolve artificial metalloenzymes for enantioselective $\mathrm{C}-\mathrm{C}$ bond forming reactions. Reaction of a diazoalkanoate with (M-PIX) affords the corresponding $\left(\mathrm{RO}_{2} \mathrm{CR}=\mathrm{M}-\mathrm{PIX}\right)$. If the substrate possesses an orthoalkylether moiety, an intramolecular $\mathrm{C}-\mathrm{H}$ insertion occurs to afford the corresponding enantioenriched dihydrobenzofurans, Scheme 3a. Addition of an alkene to $\left(\mathrm{RO}_{2} \mathrm{CR}=\mathrm{M}-\mathrm{PIX}\right)$ leads to the formation of enantio- and diastereoenriched cyclopropanes, Scheme 3b.

Exploratory screens resulting from varying the metal moiety as well as the hemoprotein lead to the identification of $\{\mathrm{IrMe}\}$ and myoglobin as the most promising combination. Genetic optimization of the catalytic performance was undertaken next. The proximal His93, originally bound to Fe-PIX was mutated, introducing either coordinating or non-coordinating amino acids. This lead to the identification of His93Ala and His93Gly as the most promising mutants. Subsequent rounds of iterative mutagenesis were aimed at tailoring the substrate binding site above the porphyrin plane. For this purpose, the distal His64 as well as positions Leu32, Phe33, Phe43, Val68, His97 and Ile99 were mutated with a focused library including hydrophobic and uncharged residues. In total, nearly five hundred (MeIr-PIX) - Mb mutants were screened. Selected results for the $\mathrm{C}-\mathrm{H}$ insertion as well as the cyclopropanation are summarized in Scheme 3 a and $b$ respectively.

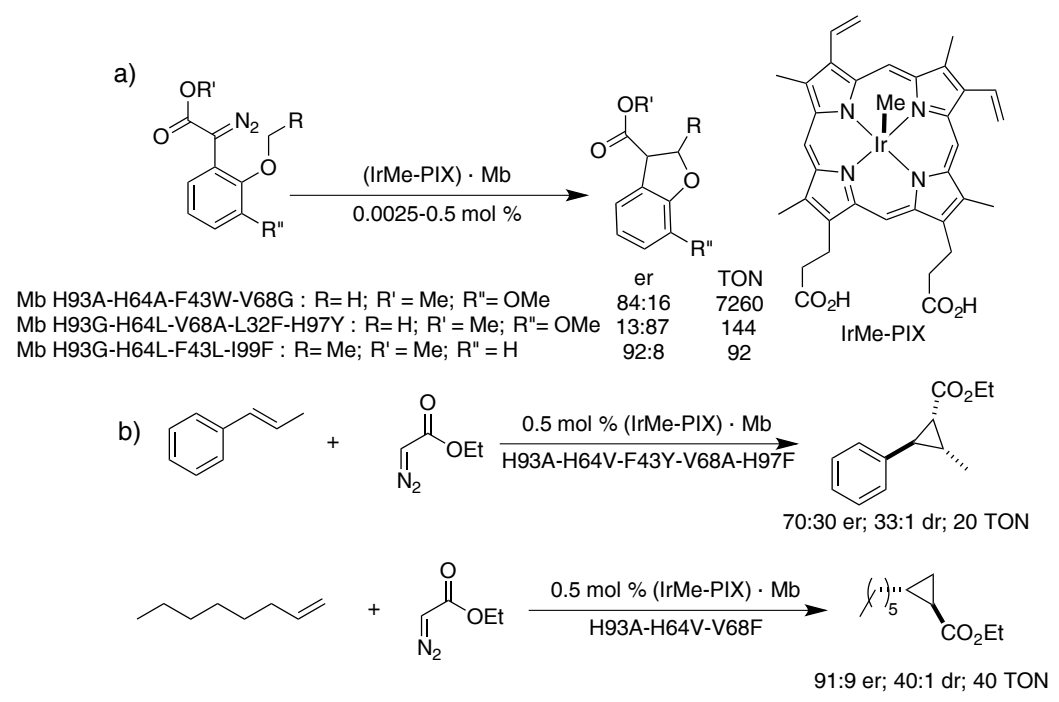

Scheme 3 Iron substitution in myoglobin $(\mathrm{Mb})$ by platinum group metals affords versatile metalloenzymes (MeIr-PIX) - Mb for $\mathrm{C}-\mathrm{C}$ bond forming reactions a) and cyclopropanation of challenging substrates $\mathrm{b}$ ). 
The contribution by Hartwig and coworkers presents several fascinating features which are highlighted below.

i) In order to speed up the screening protocol, ultimately leading to the directed evolution of (MeIr-PIX) - Mb, Escherichia coli was grown under iron-depleted conditions at low temperatures. This allowed the straightforward isolation of apo-Mb mutants, which were complemented by the metallated M-PIX cofactor. Parallel purification was achieved by affinity chromatography on a Ni(NTA) column (NTA = Nitrilotriacetate). This strategy thus allows to combine the rich chemistry of abiological PIX variants with the power of directed evolution in vitro.

ii) Thanks to the position of the "artificial" cofactor (MeIr-PIX) within myoglobin's natural active site, the abiotic substrate is completely embedded within a protein cavity. This contrasts with other anchoring strategies, whereby the cofactor is often more exposed to the solvent, thus offering significantly less control over second coordination sphere interactions between the substrate and the host protein. ${ }^{[2-4]}$ The use of focused Mb-libraries, bearing mutations in the vicinity of (MeIr-PIX) cofactor, allowed to tailor the active site to catalyse two enantioselective reactions, for which no known natural enzyme has been reported to date.

iii) Starting from an active but an essentially non-selective (MeIr-PIX) $\cdot$ WT-Mb (WT is wildtype), both enantiomers of the benzodihydrofuran product could be accessed by directed evolution. Importantly, up to > 7'000 turnovers could be achieved. This highlights the versatility of the site isolation of the reactive Ircarbene moiety, preventing catalyst deactivation. Screening the focused $\mathrm{Mb}$ library allowed to identify promising (MeIr-PIX) $\cdot$ Mb for a variety of substrates: the ester, the arene and the alkoxy functionality could all be varied affording good enantioselectivities in most cases, Scheme 3a.

iv) Enantio- and diastereoselective cyclopropanation could be achieved for both internal vinylarenes as well as unactivated alkenes. A key asset of the optimization strategy relies on the modularity of the approach since the same (M-PIX) - Mb mutant library can be screened for a variety of different reactions, metals and substrates, eventually leading to the identification of promising ArMs for various reactions, Scheme $3 \mathrm{~b}$.

Although metal-substitution has been used in the past to generate artificial metalloenzymes, their catalytic performance for new-to-nature reactions has remained limited. This contribution unambiguously demonstrates the versatility of this strategy to create and evolve ArMs for new-to-nature reactions. In view of the vast number of heme-dependent proteins, this versatile approach offers fascinating opportunities to complement other ArM strategies (Scheme 1). It may be desirable to assemble and evolve the artficial metalloenzymes in vivo in order to screen significantly larger mutant libraries or to implement ArMs in the context of synthetic biology applications. This in vivo applicability is an essential milestone toward new-to-nature metabolism and remains to be demonstrated for the noble metal-containing ArMs.

\section{Literature cited}

[1] A. Ilie, M. T. Reetz, Isr. J. Chem. 2015, 55, 51. 
[2] T. K. Hyster, T. R. Ward, Angew. Chem. Int. Ed. 2016, 55, 7344; Angew. Chem. 2016, 128, 7468.

[3] P. Srivastava, H. Yang, K. Ellis-Guardiola, J. C. Lewis, Nat. Commun. 2015, 6, DOI: $10.1038 /$ ncomms8789.

[4] T. K. Hyster, L. Knörr, T. R. Ward, T. Rovis, Science 2012, 338, 500.

[5] W. J. Song, F. A. Tezcan, Science 2014, 346, 1525.

[6] H. M. Key, P. Dydio, D. S. Clark, J. F. Hartwig, Nat. 2016, 534, 534.

[7] K. Oohora, Y. Kihira, E. Mizohata, T. Inoue, T. Hayashi, J. Am. Chem. Soc. 2013, 135, 17282.

[8] H. Renata, Z. J. Wang, F. H. Arnold, Angew. Chem. Int. Ed. 2015, 54, 3351; Angew. Chem. 2015, 127, 3408.

[9] P. S. Coelho, E. M. Brustad, A. Kannan, F. H. Arnold, Science 2013, 339, 307.

[10] M. Bordeaux, V. Tyagi, R. Fasan, Angew. Chem. Int. Ed. 2015, 54, 1744; Angew. Chem 2015, 127, 1764.

TOC

\section{Catch-phrase: Ugrading myoglobin with iridium!}

A metal-substitution strategy affords a repurposed myoglobin for challenging cyclopropanation and intramolecular $\mathrm{C}-\mathrm{H}$ activation reactions. The performance of the iridium-loaded myoglobin (orange sphere) was improved by directed evolution of eight active site residues (yellow surface).

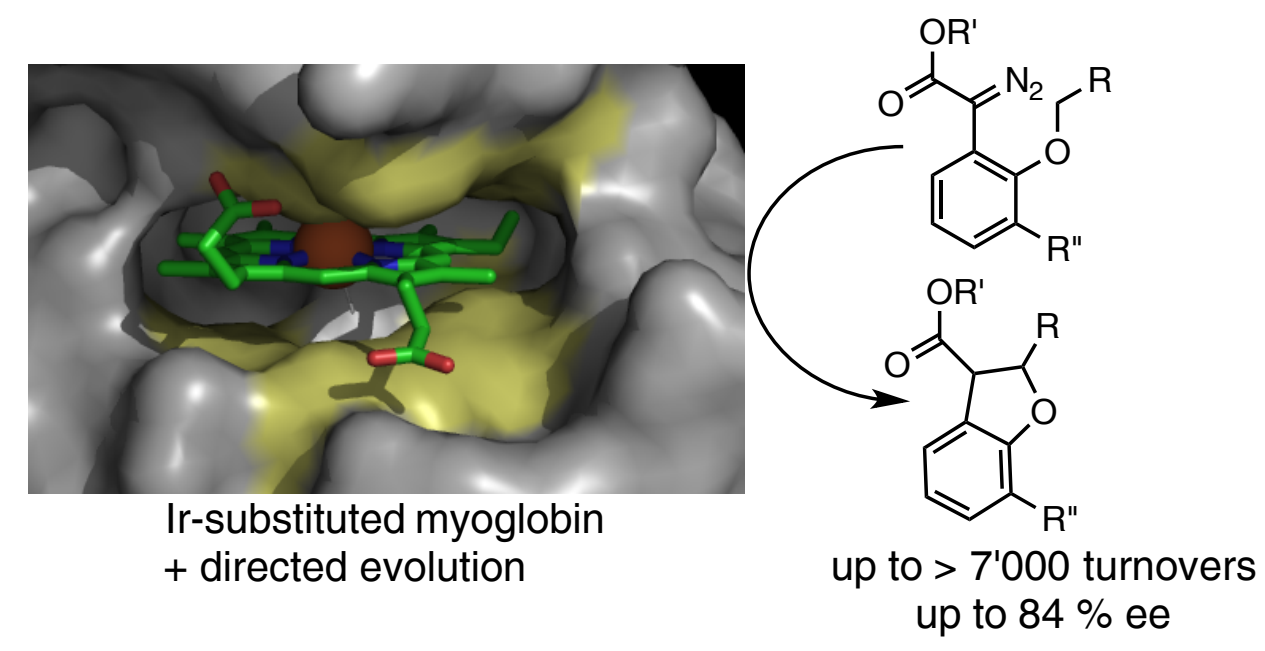

